\title{
Etude de l'évolution des insectes auxiliaires et de leurs relations avec le psylle du poirier (Psylla pyri L.) et l'acarien rouge (Panonychus ulmi Koch) dans deux vergers du Sud-Est de la France en 1979
}

\author{
Guy FAUVEL \& Pierre ATGER (*) \\ avec la collaboration de Didier COTTON \& François FAIVRE D'ARCIER
}

I.N.R.A., Laboratoire de la Chaire d'Ecologie animale et de Zoologie agricole, ENSA, Place Viala, F 34060 Montpellier Cedex.

(*) I.N.R.A., Laboratoire de Zoologie, Centre de Recherches d'Avignon, F84140 Montfavet.

\section{RÉSUMÉ}

Pyrus communis, Psylla pyri, Panonychus ulmi, Homoptera Psyllidae, Acari Tetranychidae, Lutte intégrée.

\begin{abstract}
Une étude de la répartition spatiale et de l'évolution temporelle du psylle du poirier, de l'acarien rouge et des principaux auxiliaires a été conduite par battage et contrôle visuel dans deux vergers de poirier du Sud-Est de la France en 1979. Elle montre que, vis-à-vis de Psylla pyri L., Anthocoris nemoralis F. manifeste la meilleure corrélation et la réponse numérique la plus nette. Les Orius et Orthotylus nassatus F. se montrent plus nettement liés à Panonychus ulmi Koch tandis que l'évolution des araignées traduit leur polyphagie et suit d'une manière assez marquée celle des thrips.

Un arrosage violent par aspersion perturbe beaucoup plus le développement des auxiliaires que celui du psylle et les anthocorides ont semblé plus sensibles que les mirides à un traitement à la phosalone contenant des
\end{abstract} traces de fenvalérate.

\section{SUMMARY}

Pyrus communis, Psylla pyri,

Panonychus ulmi, Homoptera Psyllidae, Acari Tetranychidac, Integrated Control.
Evolution of predaceous insects and their relationships with Psylla pyri L. and Panonychus ulmi Koch in two pear orchards of southern France in 1979.

In 1979 an experiment was set out in two pear orchards of southern France in order to appreciate the relationships between insect predators and Psylla pyri or Panonychus ulmi. Method used was the beating of 60 shoots divided into 6 or 10 subplots, fortnightly. Visual counts made on $5 \mathrm{~m}$ of wood allowed a comparison for pear psylla populations showing good similarity between the two methods regarding the general trend of evolution.

On the basis of spatial distribution inside one orchard especially and population dynamics curves, two groups appeared: one related mainly to European red mite comprised Orius sp., Orthotylus nassatus F. which is probably also phytophagous and Deraeocoris lutescens Schil. or D. serenus Doug. \& Scott. in part.

Against pear psylla, Anthocoris nemoralis F. was the only predator showing a good numerical response but others as Deraeocoris could have enhanced its regulating effect. Numerous beneficial species were met but seemed to play a minor role or appeared rather in late season as chrysopids. Araneids' numbers seemed to follow closely thrips populations.

Strong overhead sprinkling appeared disturbing the relationship between beneficial species and pear psylla. Phosalone with fenvalerate traces was more toxic for anthocorids than for mirids.

\section{INTRODUCTION}

Pour les producteurs de poires, le psylle du poirier reste un des ravageurs les plus gênants et son importance a augmenté durant ces dernières années pour des raisons qui ont été discutées ailleurs (ATGER, 1978, 1979), parmi lesquelles figure la résistance accrue aux pesticides classiques. Les nouvelles matières actives que sont les pyréthrinoïdes de synthèse ont paru prometteuses et, de fait, ont donné de bons résultats. Cependant leur emploi répété s'est rapidement traduit par des multiplications d'acariens révélatrices ici, comme dans d'autres cultures, d'excès dans les pratiques culturales notamment dans la protection phytosanitaire. Une nouvelle stratégic s'impose donc avec un allègement des programmes de lutte qui devrait être compensé par une action plus efficace des auxiliaires.

Dans le cas du psylle, la régulation des populations est largement conditionnée par le rythme de croissance de la 
plante-hôte mais l'action des ennemis naturels est loin d'être négligeable. En verger peu entretenu, les observations montrent que le psylle est rarement un problème (NiCKEL et al., 1965; VEZ, 1978) mais la conduite intensive des cultures rend le milieu très favorable au ravageur en activant la croissance des arbres et en freinant l'action des auxiliaires. Il paraît difficile de réduire considérablement la première cause pour des raisons économiques évidentes; par contre, le placement judicicux des interventions en dehors de la période d'activité des seconds a eu un effet très positif (ATGER, 1977).

Cependant, une revue de la littérature montre que si bcaucoup d'espèces sont citées comme s'attaquant aux psylles du poirier (MADSEN et al., 1963 ; BONNEMAISON, 1964 ; NICKEL et al., 1965 ; WESTIGARD et al., 1968 ; RASMY \& McPHEE, 1970), en fait, cxcepté pour l'hétéroptère Anthocoris nemoralis F, on dispose de peu de données précises pour apprécier leur incidence respective sur le développement du ravageur et même leur degré de liaison. De plus, il convient de noter qu'une grande partie des observations se rapporte au cas de Psylla pyricola Foerster, notamment en pays anglo-saxons, alors qu'en France nous

Camaret

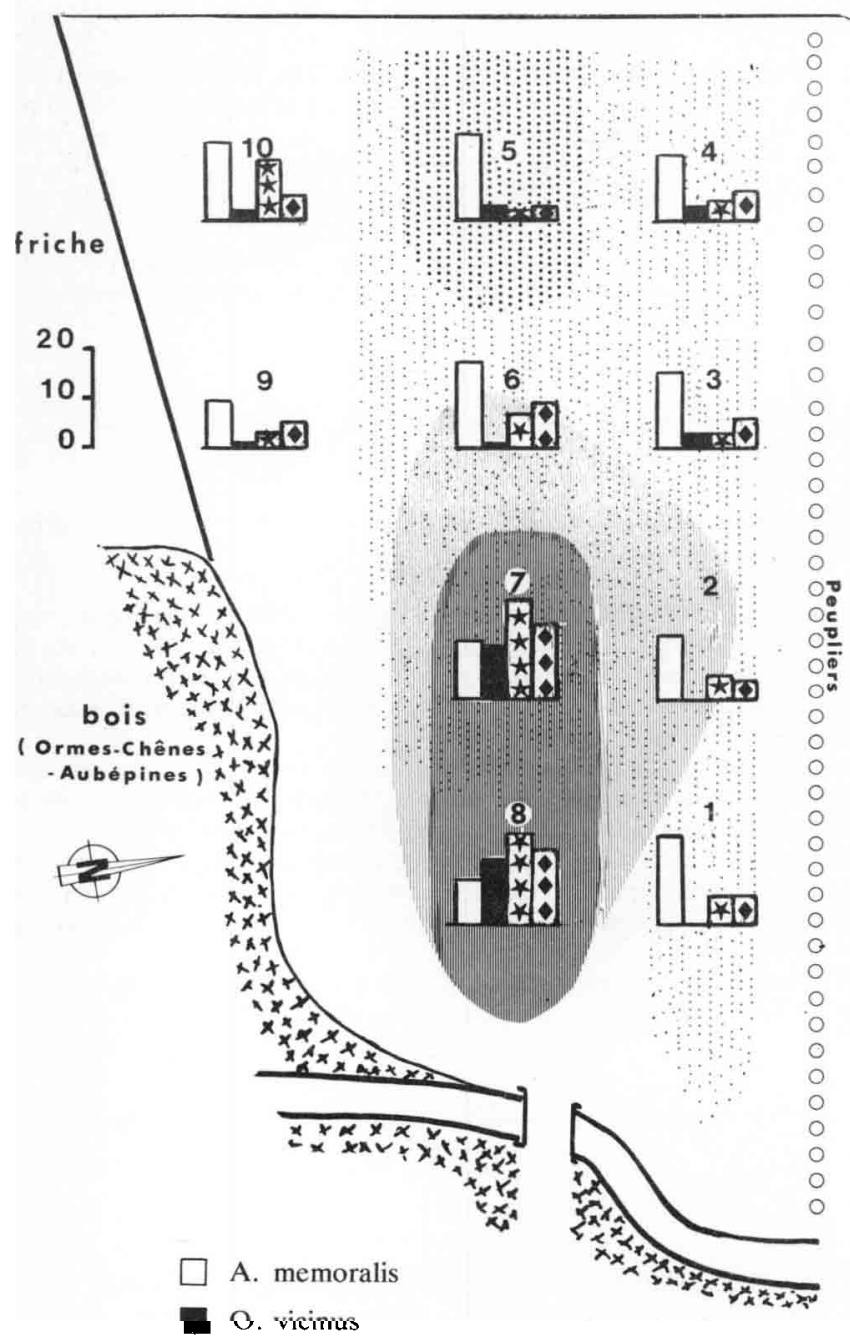

因 O. nassatus

D. lutescens, D. serenus Psylle INFESTATION FORTE Psylle INFESTATION MOYENNE P. UImI INFESTATION FORTE P. Ulmi INFESTATION MOYENNE avons affaire à $P$. pyri. C'est pour ces raisons que nous avons jugé opportun d'essayer d'analyser un peu mieux les relations entre les auxiliaires présents dans le verger et les différentes proies, l'attention étant surtout portée sur les psylles et l'acarien rouge.

Parmi les voies d'approche, l'analyse de la coïncidence spatiotemporelle peut apporter des éléments d'information sur les relations entre auxiliaires et proies, même si d'autres facteurs interviennent. Les résultats sont d'ailleurs faciles à obtenir par un plan d'échantillonnage adéquat ainsi qu'on peut le voir dans l'expérience qui sera exposée ici. Des observations ponctuelles en laboratoire apporteront les compléments nécessaires à l'interprétation.

\section{MATÉRIEL ET MÉTHODES}

\section{A. Les vergers}

Ils étaient situés près de Camaret (Vaucluse), protégés des vents du Nord par des haies de peupliers, et leur environnement ćtait assez riche en espèces végétales (cf. fig. 1). La présence des saules dans le bosquet bordant St-

St Tronquet

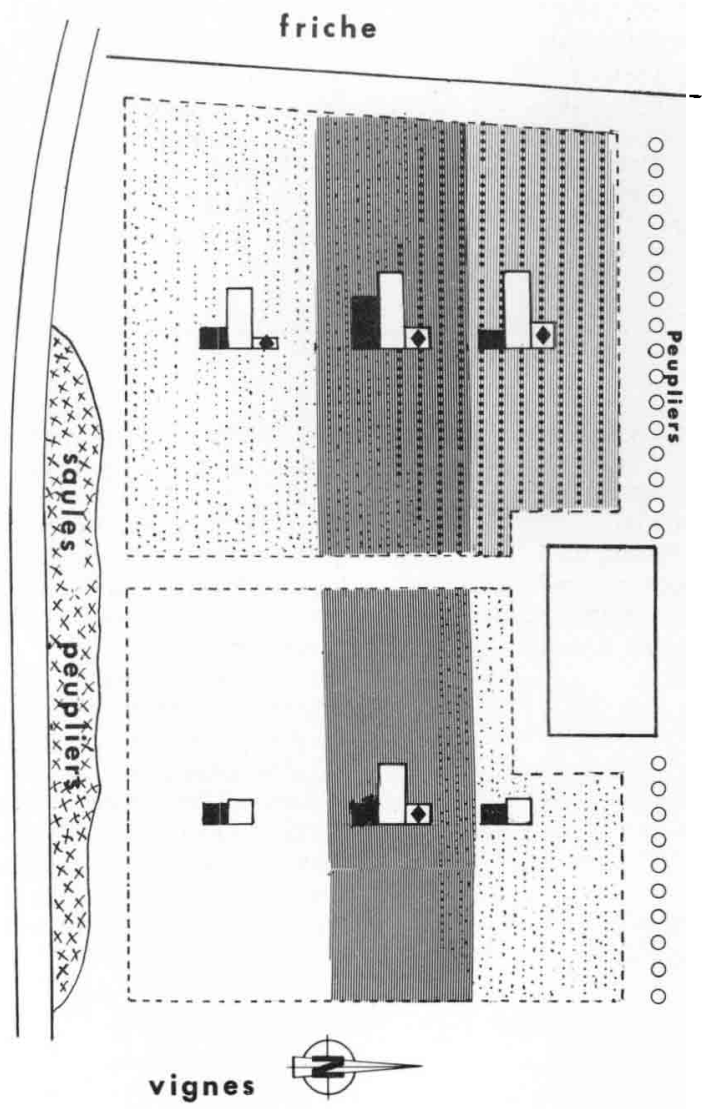

Figure 1

Répartition des différents prédateurs par rapport aux foyers de psylle et de Panonychus ulmi dans les deux vergers durant la période fin mai à mi-août 1979.

Spatial distribution of different predators against psyllid and european red mite focuses in the two orchards during the period from the end of may to mid august 1979. 
Tronquet ou de chênes, ormes, aubépines ... dans celui de Camaret était favorable à la colonisation du verger par les hétéroptères anthocorides et mirides que ces arbres hébergent fréquemment (ANDERSON, 1962, SATCHELL \& SOuTHwOOd, 1963, EHANNO, 1965, 1977).

Les poiriers, de la variété «J. GUYOT», palissés à écartement d'1,5 $\mathrm{m} \times 3 \mathrm{~m}$, avaient une hauteur de 2 à $2,5 \mathrm{~m}$.

Les programmes de traitement contre la tavelure et l'oïdium furent maintenus comme d'habitude tandis que des interventions insecticides contre le carpocapse furent effectuées en fonction des résultats du piégeage sexuel. En raison d'un nombre important de pontes hivernales de Panonychus ulmi, une application de cyhexatin fut faite en mai-juin dans chacune des parcelles. Les principales différences entre les 2 vergers sont les suivantes:

Camaret : traitement avec phosalone, contenant involontairement des traces de fenvalerate, effectué le $1^{\text {er }}$ juillet contre le carpocapse ; irrigation abondante par aspersion sur frondaison le 10 juillet pour laver le miellat sur les fruits (40 mm en $1 \mathrm{~h}$ ).

St-Tronquet : application seulement d'amitraze le 9 mai et le 10 juillet, contre psylles et acariens ; aucun traitement ne fut effectué contre le carpocapse du fait des très faibles niveaux de capture obtenus avec les pièges sexuels.

\section{B. Méthodes}

Pour la capture des auxiliaires, le battage convient bien et a été couramment utilisé. La méthodologie varie avec les auteurs, la plupart travaillant avec 50 branches battues audessus d'un entonnoir (MADSEN et al., 1963 ; BATISTE et al., 1970) ou d'une nappe d'environ $1 / 4 \mathrm{~m}^{2}$ (NICKEL et al., 1965 ;

Psylla pyri Camaret 1979

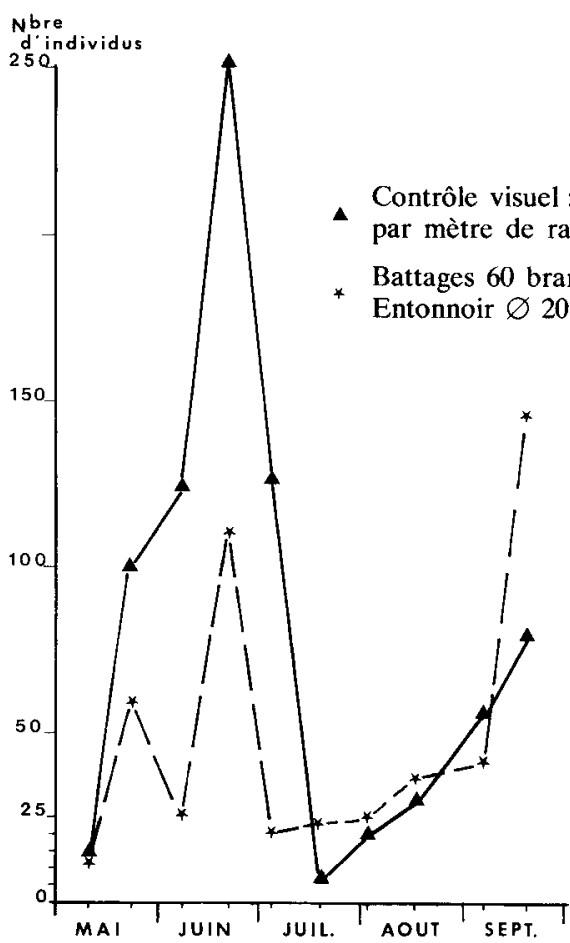

Figure 2

Courbes d'évolution des populations larvaires de psylle par battage et par contrôle dans le verger de Camaret.

Evolution of pear psylla populations according respectively to beating method and visual counts in Camaret orchard.
VEZ, 1978). Nous avons préféré un entonnoir en plastique $1 / 2$ souple de $20 \mathrm{~cm}$ de diamètre avec récolte des animaux dans l'alcool à $70^{\circ}$ ce qui permet un examen plus exhaustif des captures et une identification plus précise. En verger de pommiers, FAUVEL et al. (1981) ont montré que la précision des recensements tient davantage au nombre de branches utilisées qu'à la taille du réceptacle. Compte tenu du nombre de ces organes disponibles dans chaque parcelle élémentaire à Camaret et de façon à ne pas alourdir le travail de l'expérimentateur, nous avons retenu pour chaque verger un total de 60 rameaux répartis en 6 à 10 points, comme indiqué sur la figure 1 . Les battages eurent lieu tous les 15 jours.

Pour avoir une estimation plus précise des populations préimaginales de psylle, des comptages visuels des œufs et larves furent faits chaque semaine sur 2 rangs centraux dans la parcelle Camaret. On a pris 50 bouquets de feuilles au moment de la floraison puis un nombre variable de rameaux poussants, représentant au total à chaque observation $5 \mathrm{~m}$ de bois c'est-à-dire plus de 200 feuilles. Plus encore que pour le battage, ces valeurs sont déterminées par l'importance du travail nécessaire et il convient de souligner qu'en général les contrôles analogues effectués par les auteurs cités ci-dessus sont limités à 100 feuilles ou $20-25$ bouquets (Hoyt et al., 1978).

La comparaison des résultats de l'examen visuel des rameaux avec ceux du battage (fig. 2) montre que malgré des écarts sensibles à certains moments, la $2^{\mathrm{c}}$ méthode traduit assez bien l'évolution globale des populations de psylle et permet donc une analyse correcte des relations proie-prédateurs.

\section{RÉSULTATS}

\section{A. Répartition des ravageurs et des auxiliaires dans le verger de Camaret}

On ne s'intéressera qu'au psylle et à l'acarien rouge ainsi qu'aux groupes d'ennemis naturels les plus importants numériquement.

Le verger de Camaret s'est révélé particulièrement propice à cette partie de l'étude du fait que les foyers de psylle et de $P$. ulmi sont restés suffisamment distincts (fig. 1) ; les différentes zones ont été définies en considérant 3 niveaux d'occupation à chaque relevé et en faisant la moyenne sur 7 observations de fin mai au 16 août, les limites étant arbitrairement fixées au milieu de l'intervalle entre 2 points d'échantillonnage.

La superposition de la répartition des auxiliaires montre des coïncidences dont certaines sont déjà connues: par exemple, les Orius sont particulièrement abondants dans le foyer d'acariens, comme cela s'observe en verger de pommiers, et l'anthocoride $A$. nemoralis dans les zones où le psylle a pullulé. Deux exemplaires seulement de la coccinelle Stethorus punctillum Weise, dont on connaît la spécificité vis-à-vis des acariens, furent trouvés en 6 et 7 . Ces faibles effectifs peuvent avoir été provoqués par les traitements acaricides précoces mais la localisation de cet auxiliaire reste associée à celle de la proie.

Trois espèces d'hétéroptères mirides ont été rencontrées en assez grand nombre mais leur régime alimentaire plus étendu reste mal connu :

Deux, Deraeocoris (Knightocapsus) lutescens Schil. et $D$. (Camptobrochis) serenus Doug. et Scott., sont des prédateurs polyphages s'attaquant aux acariens et à divers insec- 
tes en verger de pommiers. BONNEMAISON (1964) cite la première espèce parmi les ennemis naturels de Psylla pyri. La troisième, Orthotylus nassatus F., vit sur les arbres à feuillage caduc et récemment HENRY (1977) a signalé qu'aux Etats-Unis cette espèce, introduite d'Europe, s'est acclimatée aux vergers de poiriers en Pennsylvanie. On constate que les maximums de population de ces 3 mirides coïncident également avec le foyer de $P$. ulmi.

La répartition dans le verger de St-Tronquet présente une bonne similitude avec celle de Camaret, mis à part quelques irrégularités comme la faiblesse relative des populations d'Orius dans la partic orientale du foyer d'acariens. Dans celle-ci, par contre, $A$. nemoralis est plus abondant que dans la zone voisine plus attaquée par le psylle. Ces anomalies n'ont pu être expliquées. On remarquera que les Deraeocoris restent plutôt en relation avec l'acarien rouge.

Naturellement ces résultats sont des indications qui doivent être contrôlées par l'étude de la dynamique des espèces en présence.

\section{B. Evolution des populations des ravageurs et des auxi- liaires}

\section{1) P. pyri et A. nemoralis}

Les figures $3 \mathrm{~A}$ et $3 \mathrm{~B}$ montrent qu'au printemps les populations de psylle ont suivi des évolutions parallèles dans les 2 vergers avec un pic à fin juin et ensuite une chute brutale du nombre de larves qui peut être due aux fortes chaleurs estivales. Il faut noter cependant que, malgré des niveaux d'adultes très voisins, le $1^{\mathrm{er}}$ pic de larves fut bien plus élevé à St-Tronquet qu'à Camaret où un traitement à la phosalone avec traces de pyréthrinoïde fut effectué le $1^{\text {er }}$ juin.

Quelques individus d' $A$. nemoralis furent trouvés en mai à Camaret mais leur multiplication ne se manifesta qu'à partir de juin (fig. 3B). On peut supposer que le traitement à la phosalone, indiqué ci-dessus, est responsable de cette absence car ensuite le développement des anthocorides fut rapide.

A partir de juillet, les situations sont devenues différentes : dans le verger de St-Tronquet s'observe une tendance constante à l'accroissement des populations d'A. nemoralis et, corrélativement, à la diminution du nombre des psylles.

Dans le second verger, les populations larvaires de $P$. pyri se développent progressivement pour culminer en septembre. Les effectifs d'A. nemoralis, initialement plus élevés qu'à St-Tronquet, déclinent considérablement jusqu'au début août. Ils s'accroîtront rapidement ensuite, plus vite d'ailleurs que le nombre de larves de psylle dans les battages. La figure $5 \mathrm{~A}$ montre cependant que la ponte du ravageur avait repris dès la mi-juillet apportant au prédateur une bonne alimentation. Cette multiplication des Anthocoris n'empêchera pas la population de larves de $P$. pyri d'atteindre à mi-septembre un niveau voisin de celui de juin.

On doit noter la réponse très nette d'A. nemoralis aux variations numériques de la proie et l'on peut penser que l'emploi du canon arroseur fut la cause d'une profonde perturbation dans l'équilibre du psylle et de l'anthocoride à Camaret. On reviendra plus loin sur ce point en considérant l'ensemble des auxiliaires (\$ III-C). Cependant, il serait sans doute exagéré d'attribuer la réduction continue des populations de psylle que l'on constate dans le verger de StTronquet à la seule action des auxiliaires. Comme on l'a rappelé en introduction, la physiologie de la plante-hôte est un facteur important de la multiplication du ravageur. L'absence d'irrigation dans cette parcelle s'est traduite en fin de saison par des symptômes nets de sécheresse et a pu contribuer fortement à la limitation des populations du phytophage.

On peut remarquer au passage que l'influence de l'application d'amitraze à St-Tronquet en juillet fut peu perceptible tant sur les Anthocoris que sur le psylle. Cette matière active est considérée comme moyennement toxique pour les auxiliaires. Par ailleurs, elle n'est efficace que sur les jeunes stades du ravageur et fut appliquée à une période où la
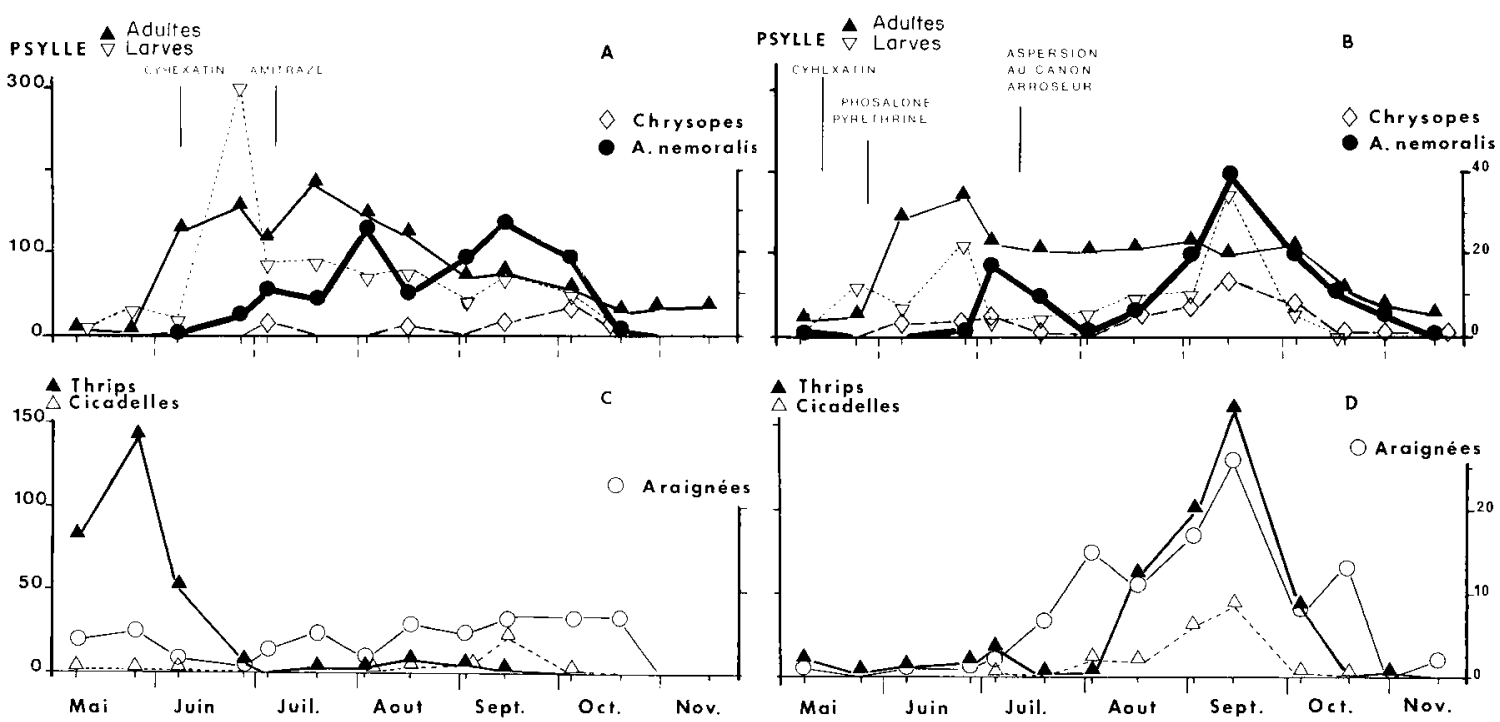

Figure 3

Evolution de différents groupes phytophages et prédateurs dans les deux vergers (nombre total d'individus pour 60 branches battues). en haut: psylle, Anthocoris nemoralis et Chrysopes en bas: thrips, cicadelles et araignées.

Evolution of different phytophagous and predaceous species in the two orchards (Total of items per 60 branches beaten). above: pear psylla, Anthocoris nemoralis and chrysopids below : thrips, leafhoppers and araneids. 

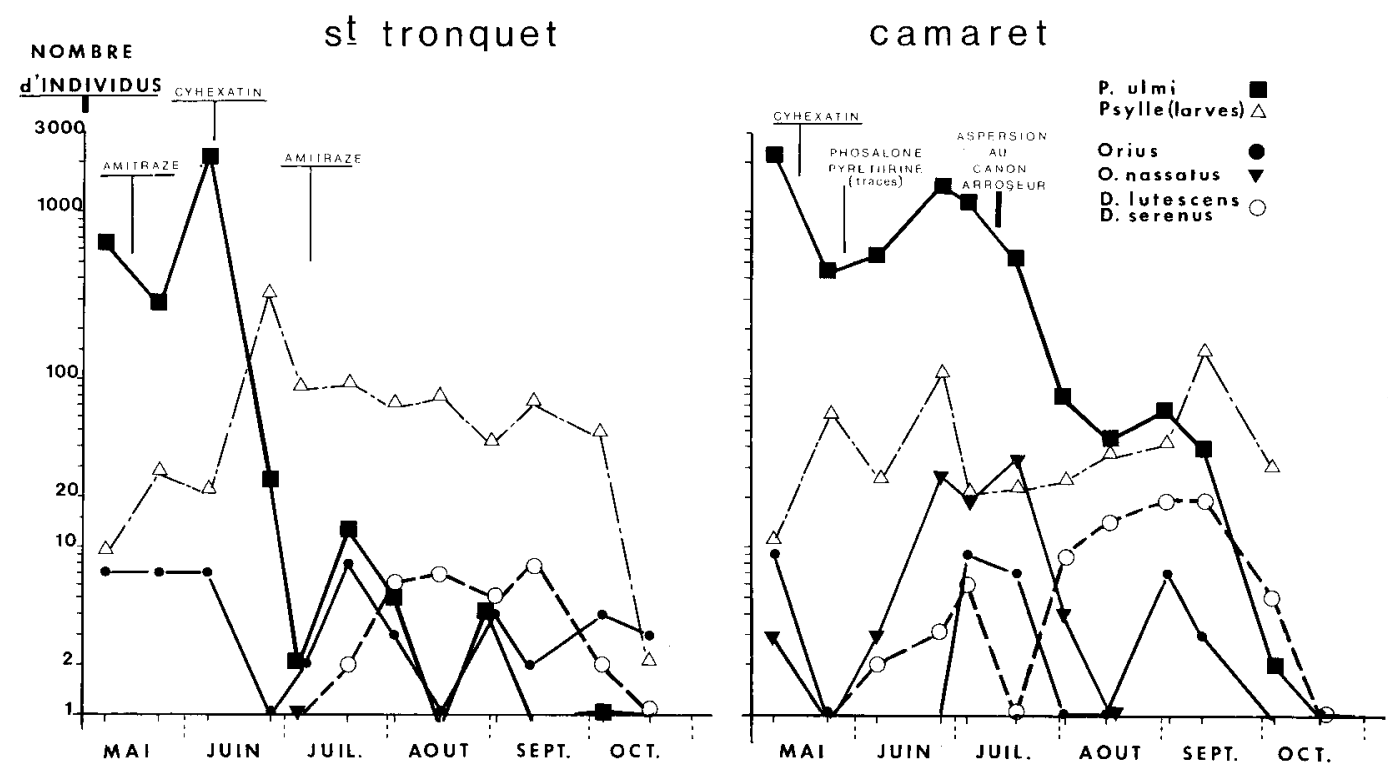

Figure 4

Courbes d'évolution des populations d'Orius, Orthotylus nassatus et Deraeocoris lutescens ou $\mathrm{D}$. serenus par rapport à celles $d u$ psylle et de l'araignée rouge (Battages de 60 branches avec entonnoir $\varnothing 20 \mathrm{~cm}$; coordonnées semilogarithmiques).

ponte des femelles diminue naturellement dans de fortes proportions.

2) Développement de Panonychus ulmi, des Orius sp. et $d$ 'Orthotylus nassatus

En raison du nombre élevé d'œufs d'hiver de $P$. ulmi, un traitement printanier était nécessaire dans les 2 parcelles et dut être fait dès le 8 mai à Camaret. Le cyhexatin se montra très efficace dans les 2 cas mais, avec l'application la plus précoce, la population résiduelle fut suffisante pour qu'une petite pullulation se produise en fin juin (fig. 4).

Parmi les espèces prédatrices présentes, on remarque particulièrement les Orius $(O$. vicinus Rib. essentiellement) dont les variations reflètent assez fidèlement celles de l'acarien rouge notamment dans le verger de St-Tronquet (fig. 4). Leur absence de fin mai à fin juin à Camaret peut être attribuée, comme pour $A$. nemorum, à l'effet du traitement phosalone + traces de fenvalérate. L'existence d'une spécificité alimentaire d' $O$. vicinus vis-à-vis de $P$. ulmi a été montrée (FAUVEL, 1971, 1972). Selon d'autres observations, elle se nourrit aussi des oufs de psylle mais l'examen de la figure 5A et $\mathrm{B}$ ne fait apparaître qu'une réponse faible et tardive à l'augmentation rapide de cette source d'alimentation à partir de mi-juillet à Camaret.

Dans ce dernier verger, une forte décroissance des effectifs de l'acarien rouge coïncide avec le développement du miride $O$. nassatus en juin-juillet (fig. 4). Cette espèce est monovoltine et les 2 pics que l'on observe sur la courbe représentent naturellement le maximum de larves puis d'adultes. Comme elle est très proche d'O. marginalis Reut., fréquente sur pommiers, on pouvait présumer que son régime alimentaire était aussi phytozoophage. La coloration violacée du tube digestif des larves récoltées semblait indiquer qu'elles se nourrissaient de $P$. ulmi et des essais en laboratoire l'ont tout à fait confirmé. Ces résultats corroborent les observations sur la répartition spatiale ( $\$$ III.A) et peuvent expliquer que cette espèce ait été presque absente à St-Tronquet. On ne doit cependant pas la considérer comme inféodée à cette proie et l'on a pu constater qu'elle
Population curves of Orius, Orthotylus nassatus and Deraeocoris lutescens or $\mathrm{D}$. serenus against psyllid or Panonychus ulmi $(60$ branches beaten, plastic funnel $20 \mathrm{~cm}$ in diam., semilogarithmic coordinates).
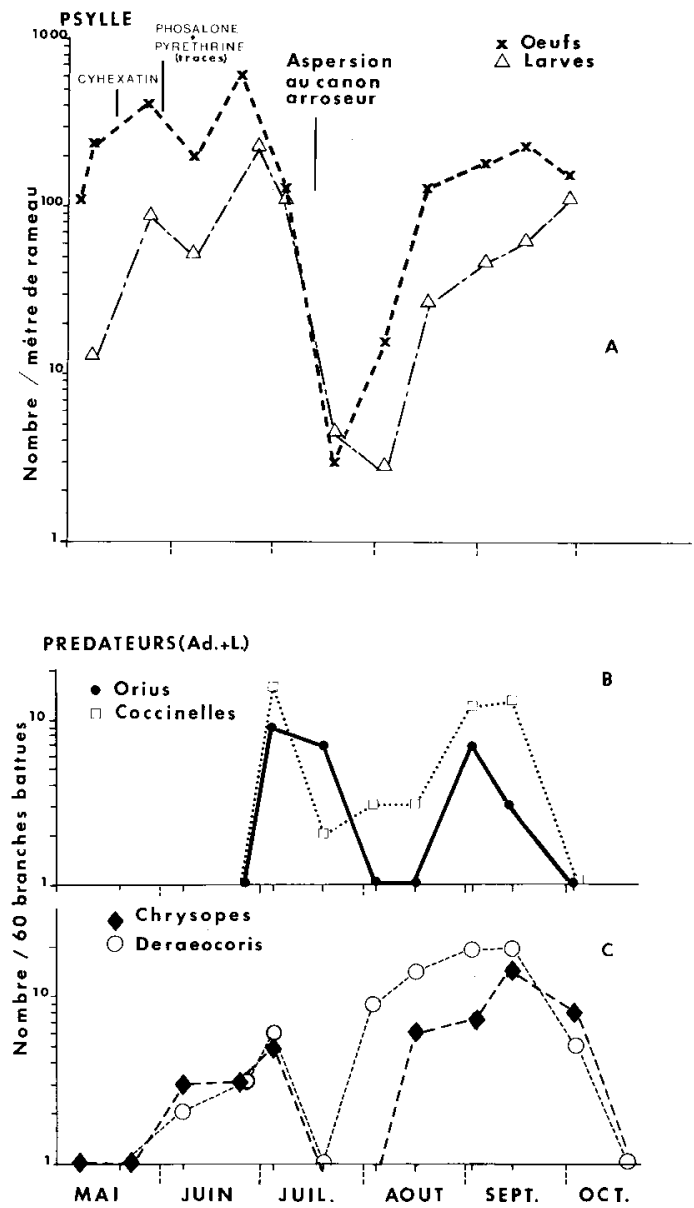

Figure 5

Evolution des différents prédateurs (Coccinelles, Chrysopes, (aeufs), Deracocoris sp. (larves), Orius sp.) par rapport aux nombres d'aufs et de larves de psylle observés par contrôle visuel dans le verger de Camaret (Coordonnées semilogarithmiques).

Evolution of different predators against the numbers of pear psylla eggs or nymphs found by visual control in Camaret orchard (semilogarithmic coordinates). 
s'attaquait aussi aux œufs et larves de psylle. Il est possible que le développement des jeunes stades soit favorisé par la présence des acariens.

Quoi qu'il en soit, en considérant l'ensemble des 2 vergers, on peut conclure qu'Orius et Orthotylus jouent un rôle plus important dans la régulation de $P$. ulmi que dans celle du psylle.

\section{3) Evolution de Deraeocoris lutescens et D. serenus (fig. 4 et 5 C)}

Ces 2 espèces très voisines étaient présentes en mélange et figurent parmi les prédateurs les plus nombreux. Selon WAGNER \& WEBER (1964, p. 49-51), elles sont polyphages, hivernent au stade adulte et la nouvelle génération de $D$. serenus apparaîtrait dès le mois de mai, avant celle de $D$. lutescens (mi-juillet). Comme on l'a rappelé au §III.A, BONNEMAISON (1964) cite cette derniếre parmi les prédateurs de $P$. pyri. A Camaret, elles ont été trouvées un peu plus tôt qu'à St-Tronquet et furent également plus nombreuses. Leur répartition spatiale est un peu semblable à celle d' $O$. nassatus et ces deux éléments feraient penser qu'elles sont liées à la présence des acariens. Cependant sur la figure 4, les fluctuations de leur population suivent seulement de façon assez lâche celles de cette proie. D'un autre côté, on ne voit pas non plus de relation très marquée avec l'évolution du psylle au stade larvaire. Par contre, un rapprochement avec la courbe de ponte de ce ravageur fait apparaître un certain parallélisme (fig. 5A et C). Il n'est donc pas impossible que la présence de l'acarien rouge soit favorable au développement de ces 2 espèces qui s'attaqueraient par ailleurs aux œufs de psylle.

\section{4) Les araignées et prédateurs divers}

Les araignées ont été considérées, dans une certaine mesure, comme des ennemis du psylle. Elles furent assez nombreuses en été dans nos vergers mais les fluctuations de la courbe de leur population, notamment à Camaret, apparaissent autant refléter celles des thrips et des cicadelles que celles du psylle (fig. 3). Ceci traduit leur polyphagie et l'on peut estimer que leur action fut faible dans les 2 vergers.

Divers coccinellides et chrysopides ont été également rencontrés et leurs effectifs ont suivi le développement de $P$. pyri (fig. 3-5). Ils ne furent abondants qu'en automne, leur nombre atteignant un peu plus d'une dizaine à Camaret contre 2 à 3 à St-Tronquet en septembre. Les larves n'ont représenté que 25 p. 100 des captures de coccinelles contre plus de 40 p. 100 pour les chrysopes.

Des mirides divers (Heterotoma merioptera Scop., Pilophorus perplexus Doug. et Scott, Campylomma verbasci Meyer-Duer, Phytocoris sp.) sont apparus dans les battages mais leur nombre fut très faible ( 7 au total) et négligeable par rapport aux autres espèces.

\section{Effet de l'aspersion effectuée au canon arroseur}

Si l'on considère les figures $3,4,5$ en ce qui concerne le verger de Camaret, on observe qu'une dépression très marquée coïncide, pour la plupart des espèces, avec l'aspersion du 10 juillet. $O$. nassatus et les Orius font exception et l'évolution de leur proie, $P$. ulmi, ne paraît que modérément affectée. Il semblerait donc que ce phénomène touche plutôt l'ensemble du psylle et de ses ennemis.

La figure 5A montre cependant que les nombres d'œufs et de larves du ravageur avaient commencé à diminuer dès la mi-juin sans doute par suite de l'arrivée des températures estivales. L'aspersion intervenant à ce moment a pu amplifier cette tendance et l'on note, sur la figure 3, que le pic d'adultes qui, à St-Tronquet, faisait suite à celui des larves, n'apparaît pas à Camaret. Il n'est pas impossible que l'arrosage ait provoqué le départ d'une partie de la population imaginale, contribuant à réduire le niveau des pontes.

La diminution des Anthocoris s'est faite progressivement durant presqu'un mois et serait donc plus liée à la raréfaction de la nourriture qu'à un effet direct de l'arrosage. Par contre, on ne peut rejeter l'hypothèse d'une influence immédiate sur la chute beaucoup plus brutale des effectifs de Deraeocoris, coccinelles et chrysopes. En effet, pour pouvoir admettre que ces espèces ont quitté spontanément et si vite le verger, il faudrait supposer qu'elles sont beaucoup plus sensibles au manque de nourriture que les Anthocoris, ce qui paraît peu probable pour l'ensemble.

Après l'aspersion, les pontes du psylle ont repris rapidement, suivies par l'apparition des larves alors que la plupart des auxiliaires réapparaissent plus tard. Il semble donc que cette pratique culturale ait eu, à terme, un effet favorable pour le ravageur du fait de la perturbation du développement des auxiliaires et d'une stimulation de la végétation des poiriers.

\section{DISCUSSION}

Cette approche spatiotemporelle des relations entre les différents auxiliaires présents dans nos vergers de poirier et les proies possibles permet donc de reconnaître des groupements fonctionnels dont la réalité est étayée par les connaissances de quelques cas précis. Elle montre le danger qu'il y a à regrouper les espèces par familles, 2 anthocorides comme les Anthocoris et les Orius ou 2 mirides comme les Deraeocoris et les Orthotylus pouvant avoir des affinités très différentes pour une même proie.

En ce qui concerne l'acarien rouge $P$. ulmi, on voit qu'il est attaqué par de très nombreux prédateurs comme dans les vergers de pommiers.

Par contre, dans notre cas, seul $A$. nemoralis a manifesté des relations vraiment étroites avec le psylle tant par sa répartition que par son évolution. Cette liaison est d'ailleurs bien connue en Europe et cette espèce a même été introduite au Canada pour cette raison (BURTS, 1971). Les autres prédateurs et notamment Deraeocoris sp. prélèvent certainement aussi leur tribut mais manifestent une polyphagie beaucoup plus grande et les variations de leurs populations répondent moins nettement à celles de ce ravageur.

Dans les études américaines ou canadiennes sur la régulation du psylle du poirier, les auxiliaires les plus fréquemment indiqués sont voisins : d'abord des anthocorides (Anthocoris et Orius) suivis par les chrysopides, les mirides du genre Deraeocoris et de nombreuses espèces de coccinelles (NICKEL et al., 1965 ; WESTIGARD et al., 1968, BATISTE et al., 1970; RASMY \& McPHEE, 1970). Les Anthocoris sont cependant beaucoup plus diversifiés selon les régions et moins nettement liés au psylle qu' $A$. nemoralis. La présence des Orius apparaît variable avec les vergers et les années et, outre le fait qu'il y a plusieurs espèces présentes, on peut regretter que très souvent on n'a pas distingué les 2 genres ni tenu compte du niveau correspondant des populations d'acariens.

Les chrysopides, bien que nettement moins abondants que les Anthocoris dans les vergers américains, sont consi- 
dérés comme des ennemis importants du psylle et, effectivement, l'évolution de leurs populations apparaît assez bien synchronisée avec celle du ravageur d'après les résultats publiés par WESTIGARD et al. (1968), BATISTE et al. (1970). Dans nos parcelles, 2 pics apparaissent dans la saison avec une dépression marquée en juillet. Il est possible que les traitements ou pratiques culturales aient eu une incidence mais il faut signaler que les travaux bioécologiques d'AL ROUECHDI et al. (1980a et b) en verger d'olivier montrent qu'il s'agit d'un phénomène constant déterminé par les périodes d'activité des différentes espèces et probablement par un effet attractif du miellat en automne. Leur action serait donc naturellement limitée en été.

Le rôle des mirides du genre Deraeocoris au sein de la faune des poiriers est encore difficile à apprécier car les observations fragmentaires dont on dispose, montrent une grande polyphagie (VIGGIANI 1971, EHANNO 1977) et nous n'avons malheureusement pas d'indications précises au sujet des espèces trouvées dans cette étude. Plusieurs auteurs indiquent qu'une espèce, $D$. brevis piceatus Knight, attaque activement $P$. pyricola au Canada (McMulLEN \& JONG 1967, RASMY \& McPHEE 1970) et des consommations de 400 œufs et larves ont été rapportées par WESTIGARD (1973). Cependant les acariens pourraient apporter un complément d'alimentation comme semblent l'indiquer les résultats obtenus par ZAVODTSHIKOVA (1974) avec $D$. (Camptobrochis) punctulatus Fall. élevé sur pucerons et tétranyques, seuls ou en mélange.

On trouve souvent une grande variété de coccinelles sur les poiriers. Pourtant leur rôle paraît modeste et nos résultats rejoignent les conclusions des auteurs anglosaxons. Quant aux araignées, d'après les observations de BATISTE et al. (1970) et les nôtres, elles n'ont que des relations lâches avec les autres éléments de la biocénose.

Notre étude montre enfin que le développement des populations d'auxiliaires peut être perturbé par différentes interventions: l'effet des traitements insecticides et la toxicité des pyréthrinoïdes de synthèse sont connus. On remarque cependant que la sensibilité apparemment plus faibles des mirides est une observation nouvelle dont l'intérêt reste à définir. La compatibilité de l'amitraze avec l'application de la lutte intégrée se confirme mais le faible impact de cette matière active sur le développement du ravageur dans le cas présent limite cet avantage.

L'effet de l'aspersion sur frondaison semble contraire aux expériences de WESTIGARD et al. (1979) mais il faut tenir compte de la différence d'intensité avec laquelle elle fut réalisée. Quoi qu'il en soit, elle paraît beaucoup plus éprouver les populations d'auxiliaires que celles du psylle.

\section{CONCLUSION}

La régulation naturelle des populations de psylle du poirier, même si elle est possible, se pose en des termes différents de celle des acariens dans la mesure où le nombre des espèces appelées à exercer une pression importante contre leur développement est bien plus réduit. En complément des hétéroptères anthocorides et mirides, il manque en particulier une espèce jouant un rôle de prédateur de nettoyage comparable à celui de la coccinelle Stethorus punctillum lors des pullulations de $P$. ulmi. Il s'ensuit que l'équilibre sera plus difficile à obtenir et que l'on devra être attentif aux perturbations apportées par les techniques culturales (taille, fertilisation, irrigation). Il sera donc important d'établir un rapport numérique initial favorable comme ATGER (1978) l'a déjà indiqué et, en même temps, d'éviter ce qui peut intensifier le développement du psylle en début de saison avant l'installation des auxiliaires. Ensuite, les interventions phytosanitaires devront être choisies et effectuées avec discernement selon les recommandations déjà faites (ATGER, 1978, 1979). Parmi les auxiliaires, $A$. nemoralis paraît le mieux répondre au développement des ravageurs et c'est donc sur lui que l'on doit porter le maximum d'attention pour favoriser son action. Compte tenu de l'évolution de ses populations comme d'ailleurs des autres auxiliaires, on voit qu'il est important d'éviter les interventions après la fin de mai ou de choisir des matières actives les épargnant.

Reçu le 2 février 1981. Accepté le 7 juillet 1981.

\section{RÉFÉRENCES BIBLIOGRAPHIQUES}

\begin{abstract}
Al Rouechdi K., Canard M., Pralavorio R., Arambourg Y., 1980a. Répartition des adultes et des pontes de Chrysopides (Neuroptera) récoltés dans une oliveraie de Provence. Neur. Int., I, 65-74.

Al Rouechdi K., Lyon J. P., Canard M., Fournier D., 1980b. Les Chrysopides (Neuroptera) récoltés dans une oliveraie du sud-est de la France. Acta Oecol. oecol. appl., 1, 173-180.

Anderson N. H., 1962. Bionomics of six species of Anthocoris (Heteroptera, Anthocoridae) in England. Trans. R. ent. Soc. London, 114, 67-94.
\end{abstract}

Atger P., 1977. Le psylle du poirier est-il un faux problème? Déf. Vég., 187, 7 pp.

Atger P., 1978. La lutte contre le psylle du poirier. Aspects nouveaux. Arboric. fruit., 288, 33-36.

Atger P., 1979. Les psylles du poirier. Biologie et contrôle en verger. Phytoma, Déf. des Cult., 311, 19-22.

Batiste W. C., Berlowitz A., Olson W. H., 1970. Evaluation of insecticides for control of codling moth on pears in California and their usefulness in an integrated control program. J. econ. Entomol., 63, 1457-62.

Bonnemaison L., 1964. Les psylles du poirier. C.R. $95^{e}$ Cong. Soc. pomol. Fr. Nîmes, 15-18 oct. Soc. pomol. Fr. éd., Villefranche, Rhône, 172-183.
Burts E., 1971. Anthocoris nemoralis. A new predator of control of pear psylla. Wash. State Hortic. Am. Proc., 67, 110-112.

Ehanno B., 1965. Notes écologiques sur les Miridae (InsectaHeteropt.) observés en Bretagne sur le chêne. Vie et Milieu, ser. C, 16, 1, 517-33.

Ehanno B., 1977. Revue faunistique des Deraeocorinae français et notes écologiques sur les espèces armoricaines (Hem.-Hét. Miridae). Ann. Soc. ent. Fr. (N.S.), 13, 117-129.

Fauvel G., 1971. Influence de l'alimentation sur la biologie d'Orius (Heterorius) vicinus Ribaut (Heteroptera, Anthocoridae). Ann. Zool. Ecol. anim., 3, 31-42.

Fauvel G., 1972. Facteurs de spécificité d'un anthocoride prédateur Orius vicinus Ribaut. Zesz. Probl. Post. Nauk Roln, 129, 251-262.

Fauvel G., Rambier A., Balduque-Martin R., 1981. La technique du battage pour la surveillance des ravageurs en culture fruitière et florale. I. Comparaison des résultats obtenus en verger de pommiers avec des entonnoirs rigides de taille moyenne et avec des entonnoirs en toile. Etude de l'influence de quelques facteurs sur l'efficacité du battage. Agronomie, 1 (2), 105-113.

Henry T. J., 1977. Orthotylus nassatus, a European plant bug new to North America (Heteroptera : Miridae). Coop. Plant Pest Rep., 2, 31, 605-608. 
Hoyt S. C., Westigard P. H., Burts E. C., 1978. Effects of two synthetic pyrethroids on the codling moth, pear psylla and various mite species in northwest apple and pear orchards. J. econ. Entomol., 71, 431-434.

Madsen H. F., Westigard P. H., Sisson R. L., 1963. Observations on the natural control of the pear psylla, Psylla pyricola Foerster, in California. Can. Entomol., 95, 837-844.

McMullen R. D., Jong C., 1967. The influence of three insecticides on the pear psylla, Psylla pyricola. Can. Entomol., 102, 1201-1208. Nickel J. L., Shimizu J. T., Wong T. T. Y., 1965. Studies on natural control of pear psylla in California. J. econ. Entomol., 58, 970-976.

Oilb/Srop, 1975. Einfuhrung in den integrierten Pflanzenschutz, Heft $\mathrm{Nr} 4$, Die Klopfmethode. Erste Auflage, PUDOC, Wageningen, $142 \mathrm{p}$

Rasmy A. H., MacPhee A. W., 1970. Studies on pear psylla in Nova Scotia. Can. Entomol., 102, 586-591.

Satchell J. E., Southwood T. R. E., 1963. The Heteroptera of some woodlands in the English Lake District. Trans. Soc. br. Entomol., 15, 117-134.

Vez F., 1978. Etude des antagonistes du psylle du poirier (Psylla piri L.) et en particulier du prédateur Anthocoris nemoralis F. dans les vergers de Suisse romande. Diss. eidg. techn. Hochsch. Zurich, $100 \mathrm{pp}$.
Viggiani G., 1971. Osservazioni biologiche sul Miride predatore Deraeocoris ruber L. (Rhynchota, Heteroptera). Boll. Lab. Entomol. agrar. Filippo Silvestri, 29, 270-286.

Wagner E., Weber H. H., 1964. Faune de Francc. 67. Hétéroptères Miridae. Féd. Franc. Soc. Sci. Nat. éd. 57, rue Cuvier Paris $\mathrm{V}^{\mathrm{c}}$, $590 \mathrm{pp}$.

Westigard P. H., 1973. The biology of and effect of pesticides on Deraeocoris brevis piceatus (Heteroptera: Miridae). Can. Entomol., 105, 1105-1111.

Westigard P. H., Gentner L. G., Berry D. W., 1968. Present status of biological control of the pear psylla in southern Oregon. J. econ. Entomol., 61, 739-743.

Westigard P. H., Lombard P. B., Allen R. B., 1979. Effects of overtree irrigation on density and damage of pear pests. J. econ. Entomol., 72, 839-840.

Zavodtshikova V. V., 1974. (Feeding, development and fecundity of Deraeocoris (Camptobrochis) punctulatus Fall. (Heteroptera, Miridae) on different diets). Entomol. Obozr., 53, 861-865. 